\title{
Beobachtung der Perseiden 1902
}

an der Sternwarte des hydrographischen Amtes der k. und k. Kriegsmarine in Pola.

Die folgende Liste enthält die Zusammenstellung der Meteore, welche in den vollkommen heiteren Nächten des 8., 9. und 10. August von zwei Beobachtern wahrgenommen wurden.

Am 1 I. August wurde die Beobachtung durch vollkommene Bewölkung vereitelt.

Summarisch entfallen auf die zehn Beobachtungsstunden 88 Sternschnuppen; davon gehören dem Sternbilde des Perseus 72 an und von diesen wurden 59 graphisch festgelegt.

Die Koordinaten des Radianten wurden für den 9. und ro. August mittelst strenger Ausgleichsrechnung gefunden.

Beobachter waren die Herren k. und k. Linienschiffsfähnrich Wilhelm Linhart und k. und k. Hauptmann Leo Dziedzicki.

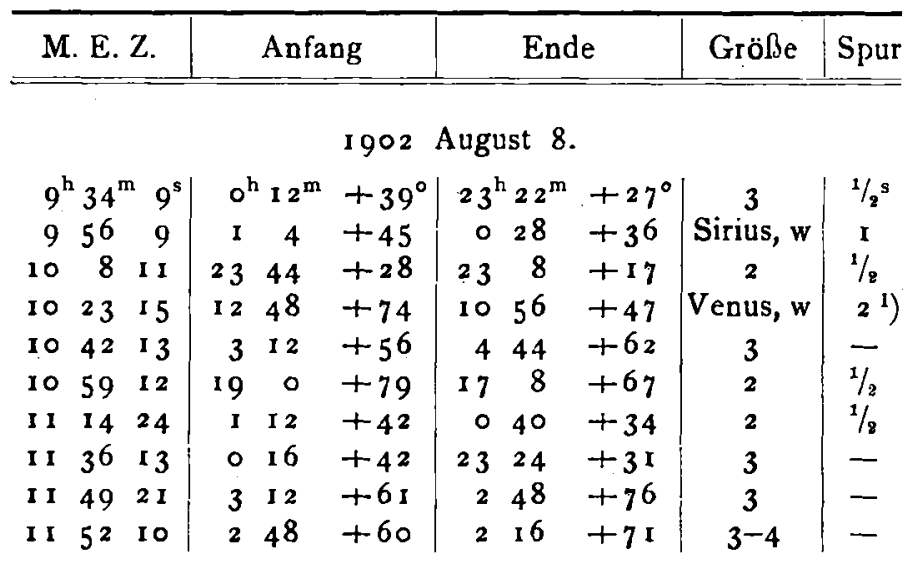

1) Langsam.

Außerdem I Perseide 4. Größe und 4 Nichtperseiden 2.-4. Größe.

1902 August 9.

\begin{tabular}{|c|c|c|c|c|c|c|c|c|c|}
\hline $0^{n}$ & I $3^{m}$ I $4^{s}$ & $23^{h}$ & $5^{6 \mathrm{~m}}$ & $+28^{\circ}$ & $23^{h}$ & I $2^{m}$ & $+17^{\circ}$ & 2 & $1 / 2^{s}$ \\
\hline 0 & 17 56 & 2 & 16 & $+5^{8}$ & $\mathbf{I}$ & 12 & +49 & $3-4$ & - \\
\hline & 2 I 4 I & 7 & $5^{6}$ & +72 & 10 & I 6 & +64 & $2-3$ & - \\
\hline & $44 \quad 14$ & $\mathbf{I}$ & 48 & +37 & I & 12 & +29 & 2 & $1 / 2$ \\
\hline & $595 \mathrm{I}$ & 3 & 0 & $+5^{8}$ & 4 & 24 & +59 & 3 & - \\
\hline & I 84 I & 2 & 4 & +50 & I & 20 & +44 & 2 & I \\
\hline & 391 & 2 & 56 & $+6 I$ & 4 & $4^{8}$ & +70 & $2-3$ & $\overline{1}$ \\
\hline & $5^{2} 36$ & I & 56 & +28 & I & 44 & $+2 I$ & 2 & $1 / 2$ \\
\hline & 226 & 2 & 8 & +63 & 0 & 4 & +72 & $3-4$ & - \\
\hline & 336 & 2 & 40 & +49 & 2 & 56 & +42 & 3 & $\overline{1}$ \\
\hline & 7 & 4 & 8 & +53 & 4 & 56 & +48 & 2 & $1 / 2$ \\
\hline & 36 & 3 & 20 & +36 & 3 & $3^{6}$ & +26 & 3 & - \\
\hline & 5036 & 2 & 4 & +39 & 2 & 18 & +24 & 3 rot & - \\
\hline & 5135 & 4 & $4^{8}$ & +50 & 6 & 12 & +39 & I w. & $1 \%$ \\
\hline & 166 & 3 & 8 & +37 & 5 & 4 & +55 & 3 & - \\
\hline & $185^{8}$ & 2 & 33 & +50 & 2 & 32 & +36 & 3 & - \\
\hline
\end{tabular}

Außerdem 10 Perseiden 2.-4. Größe und 9 Nichtperseiden 3.-4. Größe.

Radiant: $\alpha=2^{\mathrm{h}} 3^{2^{\mathrm{m}}} \delta=+566^{\circ}$.

Pola, 1902 Aug. 6.

\begin{tabular}{|c|c|c|c|c|c|c|c|c|c|}
\hline \multicolumn{2}{|c|}{ M. E. Z. } & \multicolumn{3}{|c|}{ Anfang } & \multicolumn{3}{|c|}{ Ende } & Größe & Spur \\
\hline \multicolumn{10}{|c|}{ I 902 August 10} \\
\hline $9^{h} 34$ & $8^{s}$ & $12^{h}$ & $12^{\mathrm{m}}$ & $+5^{1^{\circ}}$ & $13^{h}$ & $40^{m}$ & $+47^{\circ}$ & I gr. w. & $1 / 2^{3}$ \\
\hline 1014 & 53 & 0 & 24 & +29 & 0 & 8 & +17 & 3 & - \\
\hline 1017 & 5 & 2 & $5^{2}$ & +44 & 2 & 42 & +28 & 2 & $1 / 2$ \\
\hline 10 28 & 20 & 4 & 40 & +85 & 13 & 24 & +85 & 2 & $1 / 8$ \\
\hline I I 3 & 15 & 2 & 36 & +55 & I & 12 & +62 & 3 & - \\
\hline I 14 & 30 & 4 & 46 & +42 & 3 & 40 & +45 & 2 & - \\
\hline I I I 3 & I 7 & 4 & 44 & +54 & 6 & 24 & +57 & 2 & $1 / 8$ \\
\hline II 16 & 6 & 2 & 40 & +48 & 2 & 44 & +40 & 4 & - \\
\hline I I 20 & 50 & I & 40 & +59 & 23 & $3^{8}$ & +63 & I w. & I \\
\hline I $1 \quad 27$ & 53 & 2 & 40 & +48. & I & $3^{I}$ & +43 & $3-4$ & - \\
\hline II 31 & I 5 & 3 & IO & $+3^{6}$ & 3 & 24 & $+2 I$ & I w. & I \\
\hline I I 43 & 25 & 3 & 16 & +52 & 5 & I & $+6_{3}$ & 3 & - \\
\hline I I 44 & 47 & 2 & 56 & +55 & 3 & 48 & +69 & 2 & - \\
\hline I I 56 & 45 & 2 & 10 & $+3^{1}$ & $\mathrm{I}$ & 49 & +23 & 2 & - \\
\hline I I 59 & 46 & 2 & 32 & +43 & $\mathbf{I}$ & 34 & +32 & I & $\mathbf{I}$ \\
\hline I $2 \quad 2$ & 54 & 9 & 12 & $+5^{8}$ & I I & $4^{8}$ & +45 & I & I \\
\hline I 29 & $5^{6}$ & 3 & 28 & $+4 r$ & 4 & 4 & +28 & 3 & - \\
\hline 12 I 2 & 54 & 3 & 18 & +39 & 3 & 46 & $+26^{\circ}$ & $3-4$ & $\rightarrow$ \\
\hline 12 I 5 & 5 & 2 & 22 & +28 & 2 & 7 & +19 & I & $1 / 2$ \\
\hline 1230 & 20 & 3 & 8 & +39 & 3 & 34 & +27 & 2 & $1 / 2$ \\
\hline 1232 & 5 & 3 & 4 & +55 & 7 & 48 & +70 & I & I \\
\hline 1234 & 9 & 2 & 28 & +28 & 2 & 12 & +17 & 3 & - \\
\hline I 238 & 58 & 3 & 13 & $+39^{\circ}$ & 3 & 30 & +28 & 2 & - \\
\hline 1244 & 36 & 2 & 24 & +25 & 2 & 18 & +18 & 3 & $1 / 2$ \\
\hline 1245 & 25 & I & 54 & $+47^{\circ}$ & I & 10 & +43 & 2 & - \\
\hline 1252 & 26 & 3 & $3^{\circ}$ & $+3^{2}$ & 3 & 28 & +26 & $3-4$ & - \\
\hline 1256 & 0 & 3 & 3 & +55 & 6 & 0 & +55 & $\mathbf{I}$ & - \\
\hline 1256 & I I & 2 & 54 & $+5^{6}$ & 4 & 14 & $+5^{8}$ & 3 & $1 / 2$. \\
\hline 1256 & 54 & 3 & 48 & +30 & 4 & 0 & +23 & 2 & - \\
\hline I 39 & 46 & 3 & 19 & +24 & 3 & 20 & +10 & Sirius & - \\
\hline 1319 & 10 & 3 & 6 & $+54^{\circ}$ & 4 & I 2 & $+5^{2}$ & 2 & $1 / 2$. \\
\hline 1325 & 6 & 2 & $5^{8}$ & $+43^{\circ}$ & 3 & 40 & +31 & 3 & - \\
\hline I3 30 & 49 & 2 & 40 & +29 & 2 & 32 & +21 & $3-4$ & - \\
\hline
\end{tabular}

Außerdem 2 Perseiden 3.-4. Größe und 3 Nichtpera seiden 2.-4. Größe.

Radiant: $\alpha=3^{\mathrm{h}} 2^{\mathrm{m}} \delta=+54^{\circ} .5$.

Der Vorstand der Sternwarte: Koss.

\section{Berichtigung zu den Wiener Annalen Bd. V.}

In dem von Prof. E. Weiß in den Wiener Annalen Bd. V pp. 24 ff. mitgeteilten »Unterschied der Reduktionstafeln fur Lalandes Histoire Céleste von Hansen und Nissen und von v. Asten ist auf p. I 29 für H. C. Seite 1 r r I794 Aug. r6. $Z=53^{\circ}$ der Wert für $\Delta p$ falsch angegeben. Für denselben ist zu lesen: $\Delta p==+0.2^{\prime \prime}-0 d$.

Straßburg E., 1902 Aug. 2.

B. Cohn. 